\section{Calix[4]arene Salenes: A Bifunctional Receptor for $\mathrm{NaH}_{2} \mathrm{PO}_{4}$}

\author{
Dmitry M. Rudkevich, Willem Verboom, and \\ David N. Reinhoudt \\ Laboratory of Organic Chemistry, University of Twente, \\ P.O. Box 217, 7500 AE Enschede, The Netherlands
}

Received February 14, 1994

\section{Introduction}

Calix[4]arenes are important building blocks in supramolecular chemistry. ${ }^{1,2}$ They can be selectively functionalized both at the phenolic $\mathrm{OH}$ groups (lower rim) and at the para positions of the phenol rings (upper rim). ${ }^{3}$ The calixarene platform provides unique possibilities to organize several binding sites in an array complementary to a potential guest. Selective calixarene-based receptors for cations ${ }^{4}$ and neutral molecules ${ }^{5}$ have been synthesized in the past decade. Very recently the first representatives of calixarene-containing anion receptors have been reported. ${ }^{6}$

Previously we reported that neutral metalloclefts and metallomacrocycles containing both an immobilized Lewis acidic $\mathrm{UO}_{2}$-center and amido $\mathrm{C}(\mathrm{O}) \mathrm{NH}$ units as additional binding sites are excellent receptors for anions with a high selectivity for dihydrogen phosphate $\mathrm{H}_{2} \mathrm{PO}_{4}{ }^{-7}$. In the present paper we report, in addition to the synthesis of a new representative of a $\mathrm{UO}_{2}$-containing anion receptor based on a calix[4]arene, the first example of a neutral calix[4]arene-based bifunctional receptor ${ }^{8}$ which contains

(1) Gutsche, C. D. Calixarenes, Monographs in Supramolecular Chemistry; Stoddart, J.F., Ed.; The Royal Society of Chemistry: London, 1989 ; Vol. 1.

(2) Vicens, J.; Böhmer, V. Calixarenes: A Versatile Class of Macrocyclic Compounds; Topics in Inclusion Science; Kluwer Academic Press: Dordrecht, 1991; Vol. 3.

(3) (a) van Loon, J.-D.; Verboom, W.; Reinhoudt, D. N. Org. Prep. Proc. Int. 1992, 24, 437. (b) van Dienst, E.; Iwema Bakker, W. I. Engbersen, J. F. J.; Verboom, W.; Reinhoudt, D. N. Pure Appl. Chem. $1999,65,387$.

(4) For recent examples on cation recognition by calixarene based receptors see: (a) Shinkai, S.; Fujimoto, K.; Otsuka, T,; Herman, H. L. J. Org. Chem. 1992, 57, 1516. (b) Arnaud-Neu, F.; Barrett, G.; Harris, S. J.; Owens, M.; McKervey, M. A.; Schwing-Weill, M.-J.; Schwinte, P. Inorg. Chem. 1993, 32, 2644. (c) Brzozka, Z.; Lammerink, B.; Reinhoudt, D. N.; Ghidini, E.; Ungaro, R. J. Chem. Soc., Perkin Trans. 2 1993, 1037. (d) Beer, P. D.; Martin, J. P.; Drew, M. G. B. Tetrahedron 1992, 48, 9917. (e) Cobben, P. L. H. M.; Egberink, R. J. M.; Bomer, J. G.; Bergveld, P.; Verboom, W.; Reinhoudt, D. N. J. Am. Chem. Soc. 1992, 114,10573 .

(5) For recent examples of recognition of neutral molecules by calixarene-based hosts see: (a) Gutsche, C. D.; See, K. A. J. Org. Chem. 1992, 57, 4527. (b) van Loon, J.-D.; Janssen, R. G.; Verboom, W. Reinhoudt, D. N. Tetrahedron Lett. 1982, 33, 5125. (c) Murakami, H.; Shinkai, S. J. Chem. Soc., Chem. Commun. 1993, 1533.

(6) (a) Beer P. D. Dickson, C. A P. Fletcher, N. Goulden, A J. Grieve, A.; Hodacova, J.; Wear, T. J.Chem. Soc., Chem. Commun. 1993, 828. (b) Morzherin, Y.; Rudkevich, D. M.; Verboom, W.; Reinhoudt, D. N. J. Org. Chem. 1993, 58, 7602. (c) Beer, P. D.; Chen, Z.; Goulden, A. J.; Graydon, A.; Stokes, S. E.; Wear, T. J. Chem. Soc., Chem. Commun. 1993,1834 .

(7) (a) Rudkevich, D. M.; Stauthamer, W. P. R. V.; Verboom, W.; Engbersen, J. F. J.; Harkema, S.; Reinhoudt, D. N. J. Am. Chem. Soc. 1992, 114, 9671. (b) Rudkevich, D. M.; Verboom, W.; Brzozka, Z.; Palys, M. J.; Stauthamer, W. P. R. V.; van Hummel, G. J.; Franken, S. M.; Harkema, S.; Engbersen, J. F. J.; Reinhoudt, D. N. J. Am. Chem. Soc., in press.

(8) Only a few examples of macrocyclic bifunctional receptors are known: (a) Schmidtchen, F.P. J. Org. Chem. 1986, 51, 5161. (b) Reetz, M. T.; Niemeyer, C. M.; Harms, K. Angew. Chem., Int. Ed. Engl. 1991, 30, 1472. (c) Rudkevich, D. M.; Brzozka, Z.; Palys, M.; Visser, H. C. Verboom, W.; Reinhoudt, D. N. Angew. Chem., Int. Ed. Engl. 1994, 33, 467.

$0022-3263 / 94 / 1959-3683 \$ 04.50 / 0$ both anionic and cationic binding sites and is able to complex simultaneously anionic and cationic species.

\section{Results and Discussion}

The synthesis of receptors 8a,b is depicted in Scheme 1. Calix[4]arene diester 1 was prepared by alkylation of unsubstituted calix[4]arene ${ }^{9}$ with ethyl bromoacetate in the presence of 1 equiv of potassium carbonate as a base in refluxing acetonitrile in $88 \%$ yield. Nitration of 1 with $65 \% \mathrm{HNO}_{3}$ in a mixture of acetic acid and $\mathrm{CH}_{2} \mathrm{Cl}_{2}$ gave the dinitrocalix [4]arene 2 in $51 \%$ yield with the expected ${ }^{10}$ selectivity on the more reactive phenol unit of 1 . Alkylation of $\mathbf{2}$ with ethyl bromoacetate and sodium carbonate as a base in refluxing acetonitrile afforded tetraester 3 in $70 \%$ yield. The ${ }^{1} \mathrm{H}$ NMR spectrum of 3 shows only two doublets $(4.93$ and $3.35 \mathrm{ppm}, J=13.9 \mathrm{~Hz}$ ) for the methylene bridge protons which proves the "cone" conformation of the calix[4]arene skeleton. Subsequent reduction of 3 with $\mathrm{SnCl}_{2} \cdot 2 \mathrm{H}_{2} \mathrm{O}$ in refluxing ethanol gave the corresponding diaminocalix[4]arene $\mathbf{4 b}$ in $55 \%$ yield.

Reaction of 1,3-diaminocalix[4]arenes $4 \mathbf{a},{ }^{11} \mathbf{b}$ with chloroacetyl chloride in the presence of $\mathrm{Et}_{3} \mathrm{~N}$ in $\mathrm{CH}_{2} \mathrm{Cl}_{2}$ gave the corresponding 1,3-bis(chloroacetamido)calix[4]arenes $5 \mathbf{a}, \mathbf{b}$ in 69 and $64 \%$ yields, respectively. Bisaldehydes $6 a, b$ were obtained by alkylation of 2-(2-allyloxy)-3hydroxybenzaldehyde ${ }^{12}$ with $\mathbf{5 a}, \mathbf{b}$ in the presence of potassium carbonate in 59 and $64 \%$ yields, respectively. Subsequent palladium-catalyzed deallylation ${ }^{13}$ of calixarenes $6 \mathbf{a}, \mathbf{b}$ afforded bisaldehydes $7 \mathbf{a}, \mathbf{b}$ in quantitative yield which were used without purification for the cyclization step.

Reaction of bisaldehydes 7a,b with cis-1,2-diaminocyclohexane ${ }^{14}$ and $\mathrm{UO}_{2}(\mathrm{OAc})_{2} \cdot 2 \mathrm{H}_{2} \mathrm{O}$ in refluxing ethanol under high dilution conditions gave the receptors $8 \mathbf{a}, \mathbf{b}$ which were isolated in 9 and $15 \%$ yields, respectively, after column chromatography. The moderate yields of compounds 8a,b compared with known $\mathrm{UO}_{2}$-containing metallomacrocycles ${ }^{12,15}$ may be explained by the lack of a suitable template in the cyclization step. The absorptions in the ${ }^{1} \mathrm{H}$ NMR spectra at 9.34 and $9.48 \mathrm{ppm}$ and in the IR spectra at 1615 and $1617 \mathrm{~cm}^{-1}$ for compounds 8a and $8 b$, respectively, proved imino bond formation. The presence of the $\mathrm{UO}_{2}$ moiety is in agreement with the uranium-oxygen vibrations in the IR spectra at 895$905 \mathrm{~cm}^{-1}$. Because of the "cone" conformation of the calix[4]arene unit in the ${ }^{1} \mathrm{H}$ NMR spectra there are only two doublets (4.30 and $3.12 \mathrm{ppm}$ for $8 \mathrm{a}$ and 4.80 and $3.19 \mathrm{ppm}$ for $8 b$ ) for the methylene bridge protons.

Compounds 8a,b both contain the combination of a $\mathrm{UO}_{2}$-Lewis acidic center and $\mathrm{C}(\mathrm{O}) \mathrm{NH}$ groups which is known to act as an anionic binding site. ${ }^{7}$ In addition,

(9) Gutsche, C. D.; Lin, L.-G. Tetrahedron 1986, 42, 1633.

(10) van Loon, J.-D.; Arduini, A.; Coppi, L.; Verboom, W.; Pochini, A.; Ungaro, R.; Harkema, S.; Reinhoudt, D. N. J. Org. Chem. 1990, 55, 5639.

(11) Timmerman, P. Thesis, University of Twente, 1994.

(12) van Staveren, C. J.; van Eerden, J.; van Veggel, F. C. J. M. Harkema, S.; Reinhoudt, D. N. J. Am. Chem. Soc. 1988, 110, 4994.

(13) Yamada, T.; Goto, K.; Mitsuda, Y.; Tsuji, J. Tetrahedron Lett. $1987,28,4557$.

(14) We have chosen the cis isomer because in the chair conformer of the cyclohexyl moiety one nitrogen atom is located in the equatorial and another in the axial position. According to a CPK model, the nitrogens in these positions give after cyclization the desired cavity for immobilization of the uranyl cation.

(15) van Doorn, A. R.; Schaafstra, R.; Bos, M.; Harkema, S.; van Eerden, J.; Verboom, W.; Reinhoudt, D. N. J. Org. Chem. 1991, 56, 6083.

1994 American Chemical Society 
Scheme 1
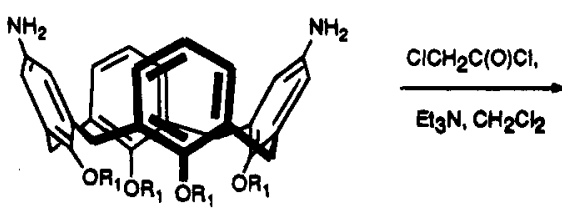

4a: $\mathrm{R}_{\mathbf{l}}=\mathrm{n}-\mathrm{Pr}$

4b: $\mathrm{R}_{1}=\mathrm{CH}_{2} \mathrm{C}(\mathrm{O}) \mathrm{OEt}$

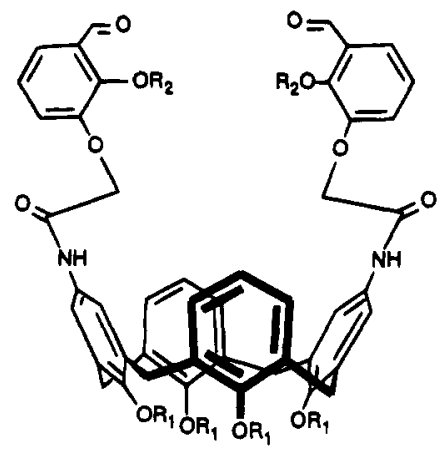

6a: $R_{1}=n-P r, R_{2}=A l l$

6b: $\mathrm{R}_{1}=\mathrm{CH}_{2} \mathrm{C}(\mathrm{O}) \mathrm{OEL}, \mathrm{R}_{2}=$ All

7a: $R_{1}=n \cdot P r, R_{2}=H$

7b: $\mathbf{R}_{1}=\mathrm{CH}_{2} \mathrm{C}(\mathrm{O}) \mathrm{OEt}, \mathrm{R}_{2}=\mathbf{H}$

Chart 1

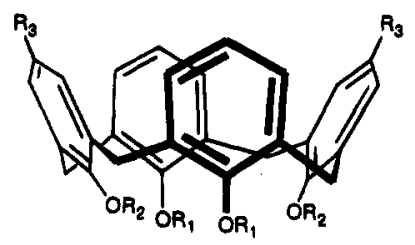

$$
\begin{aligned}
& \text { 1: } \mathrm{R}_{1}=\mathrm{CH}_{2} \mathrm{C}(\mathrm{O}) \mathrm{OEt}, \mathrm{R}_{2}=\mathrm{R}_{3}=\mathrm{H} \\
& \text { 2: } \mathrm{R}_{\mathbf{1}}=\mathrm{CH}_{2} \mathrm{C}(\mathrm{O}) \mathrm{OEt}, \mathrm{R}_{2}=\mathrm{H}, \mathrm{R}_{\mathbf{3}}=\mathrm{NO}_{2} \\
& \text { 3: } \mathbf{R}_{1}=\mathrm{R}_{\mathbf{2}}=\mathrm{CH}_{2} \mathrm{C}(\mathrm{O}) \mathrm{OEt}, \mathrm{R}_{3}=\mathrm{NO}_{2}
\end{aligned}
$$

calixarene $8 \mathrm{~b}$ contains also four preorganized ester fragments which are known to complex alkali metal cations with a high selectivity for $\mathrm{Na}^{+}{ }^{16}$ A study of the binding ability of receptors $\mathbf{8 a}, \mathbf{b}$ shows that they both selectively bind dihydrogen phosphate $\mathrm{H}_{2} \mathrm{PO}_{4}{ }^{-17}$ From ${ }^{1} \mathrm{H}$ NMR dilution experiments with $\mathrm{Bu}_{4} \mathrm{~N}^{+} \mathrm{H}_{2} \mathrm{PO}_{4}^{-}$in DMSO- $d_{6}$ association constants $K_{\text {ass }}$ of $3.5 \times 10^{2} \mathrm{M}^{-1}$ and $3.9 \times 10^{2} \mathrm{M}^{-1}$ were calculated for $8 \mathrm{a}$ and $8 \mathrm{~b}$, respectively. The contribution of the $\mathrm{C}(\mathrm{O}) \mathrm{NH}-\mathrm{H}_{2} \mathrm{PO}_{4}{ }^{-}$hydrogen bond interaction to the overall anion complexation can be clearly seen even in polar DMSO- $d_{6}$ from a significant downfield shift of the $\mathrm{C}(\mathrm{O}) \mathrm{NH}$ protons of ca. $0.4 \mathrm{ppm}$ upon complexation. Only slight shifts were observed upon dilution experiments with tetrabutylammonium salts of $\mathrm{Cl}^{-}$, $\mathrm{HSO}_{4}^{-}$, and $\mathrm{ClO}_{4}^{-}$anions which indicates their weak binding $\left(K_{\text {ass }}<10 \mathrm{M}^{-1}\right.$ ).

(16) Arnaud-Neu, F.; Collins, E. M.; Deasy, M.; Ferguson, G.; Harris S. J.; Kaitner, B.; Lough, A. J.; McKervey, M. A.; Marques, E.; Ruhl, B. L.; Schwing-Weill, M. J.; Seward, E. J. Am. Chem. Soc. 1989, 111, 8681.

(17) Only weak binding was obtained for the divalent $\mathrm{HPO}_{4}{ }^{2-}$ anion.
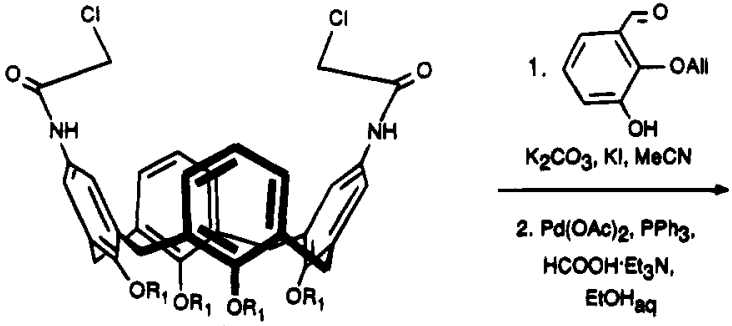

5a: $R_{1}=n-P T$

5b: $\mathrm{R}_{1}=\mathrm{CH}_{2} \mathrm{C}(\mathrm{O}) \mathrm{OEt}$
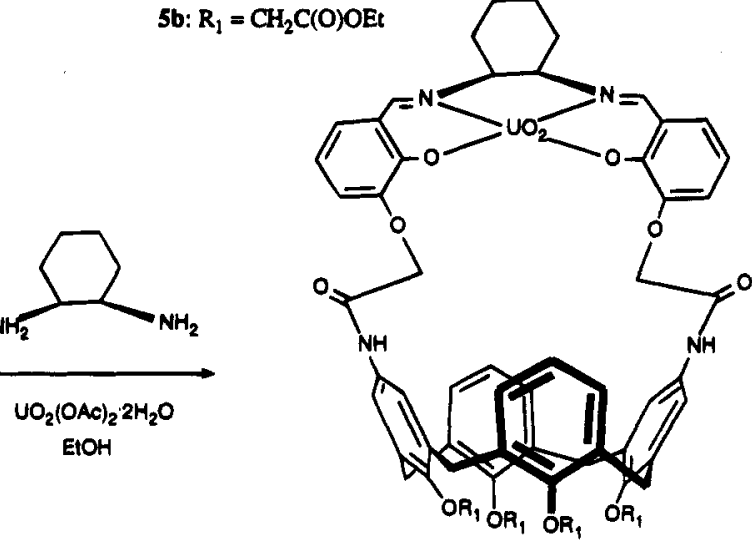

8a: $R_{1}=n-P r$

8b: $\mathrm{R}_{1}=\mathrm{CH}_{2} \mathrm{C}(\mathrm{O}) \mathrm{OEt}$
In the negative $F A B$ mass spectra of the $1: 1$ complexes of $8 \mathrm{a}$ and $\mathbf{8 b}$ with $\mathrm{Bu}_{4} \mathrm{~N}^{+} \mathrm{H}_{2} \mathrm{PO}_{4}{ }^{-}$, prepared by mixing of host and guest in $\mathrm{MeCN}$, intense peaks corresponding to $\left[8 \mathrm{a}+\mathrm{H}_{2} \mathrm{PO}_{4}^{-}\right]^{-}$and $\left[\mathbf{8 b}+\mathrm{H}_{2} \mathrm{PO}_{4}^{-}\right]^{-}$, respectively, were observed. Moreover, in the positive FAB mass spectrum of the $1: 1$ complex of $8 \mathbf{b}$ and $\mathrm{NaH}_{2} \mathrm{PO}_{4}$, prepared by mixing of host and guest in $\mathrm{MeCN}-\mathrm{H}_{2} \mathrm{O}, 10: 1$, an intense peak corresponding to $\left[8 b+\mathrm{Na}^{+}\right]^{+}$was observed, while the corresponding negative FAB mass spectrum of the same sample yielded an intense peak for $\left[8 \mathbf{b}+\mathrm{H}_{2} \mathrm{PO}_{4}^{-}\right]^{-}$, which proves the complexation of both cation and anion in one bifunctional receptor molecule.

Currently we are applying calix[4]arene-based bifunctional receptors for selective separation of alkali metal phosphates by transport through supported liquid membranes. ${ }^{18}$

\section{Experimental Section}

Melting points are uncorrected. ${ }^{1} \mathrm{H}$ NMR and ${ }^{13} \mathrm{C}$ NMR spectra were recorded in $\mathrm{CDCl}_{3}$ with $\mathrm{Me}_{4} \mathrm{Si}$ as internal standard unless stated otherwise. Fast atom bombardment (FAB) mass spectra were obtained with $m$-nitrobenzyl alcohol as a matrix. All solvents were purified by standard procedures. Petroleum ether refers to the fraction with bp $60-80^{\circ} \mathrm{C}$. All other chemicals were analytically pure and were used without further purification. Unsubstituted calix[4]arene $e^{9}$ and compound $4 a^{11}$ were prepared according to literature procedures. All reactions were carried out under an argon atmosphere.

In the workup procedures the (combined) organic layers were washed with water $(2 \times)$ and dried with $\mathrm{MgSO}_{4}$, whereupon the solvent was removed under reduced pressure. The presence of

(18) (a) Nijenhuis, W. F.; Buitenhuis, E. G.; de Jong, F.; Sudhölter, E. J. R.; Reinhoudt, D. N. J. Am. Chem. Soc. 1801, 113, 7963. (b) Nijenhuis, W. F.; van Doorn, A. R.; Reichwein, A. M.; de Jong, F.; Reinhoudt, D. N.J. Am. Chem. Soc. 1991, 113, 3607. 
solvent in the analytical samples was confirmed by ${ }^{1} \mathrm{H}$ NMR spectroscopy.

25,27-Bis[(ethoxycarbonyl)methoxy]-26,28-dihydroxy. calix [4]arene (1). A mixture of calix[4]arene ${ }^{9}(4.02 \mathrm{~g}, 9.5 \mathrm{mmol})$, $\mathrm{K}_{2} \mathrm{CO}_{3}(1.44 \mathrm{~g}, 10.4 \mathrm{mmol})$, and bromoethyl acetate $(2.1 \mathrm{~mL}, 19$ mmol) in acetonitrile $(150 \mathrm{~mL})$ was refluxed for $18 \mathrm{~h}$. After filtration the solvent was removed, and the residue was dissolved in $\mathrm{CH}_{2} \mathrm{Cl}_{2}(100 \mathrm{~mL})$ and washed with water $(2 \times 100 \mathrm{~mL})$. After evaporation of $\mathrm{CH}_{2} \mathrm{Cl}_{2}$ the crude product was recrystallized from $\mathrm{MeOH}$ to give pure 2 as a colorless solid: yield 88\%; mp 166-168 ${ }^{\circ} \mathrm{C}$ (methanol); ${ }^{1} \mathrm{H}$ NMR $\delta 7.61$ (s, $2 \mathrm{H}$ ), 7.13, 7.01 (d, $8 \mathrm{H}, J=$ $8.0 \mathrm{~Hz}), 6.82,6.78(\mathrm{t}, 4 \mathrm{H}, J=8.0 \mathrm{~Hz}), 4.81(\mathrm{~s}, 4 \mathrm{H}), 4.57,3.41$ $(d, 8 \mathrm{H}, J=13.6 \mathrm{~Hz}), 4.43(\mathrm{q}, 4 \mathrm{H}, J=7.2 \mathrm{~Hz}), 1.32(\mathrm{t}, 6 \mathrm{H}, J$ $=7.2 \mathrm{~Hz}) ;{ }^{13} \mathrm{C}$ NMR $\delta 168.9,153.0,152.4(\mathrm{~s}), 133.2,129.2,128.5$, 128.2 (d), 125.6, 119.1 (s), 72.5, 61.4, 31.5 (t), 14.2 (q); MS-FAB $m / z 597.7\left[(\mathrm{M}+\mathrm{H})^{+}\right.$, calcd 597.7]. Anal. Calcd for $\mathrm{C}_{36} \mathrm{H}_{36} \mathrm{O}_{8}: \mathrm{C}$, 72.47; $\mathrm{H}, 6.08$. Found: $\mathrm{C}, 72.35 ; \mathrm{H}, 6.00$.

25,27-Bis[(ethoxycarbonyl)methoxy]-26,28-dihydroxy-5, 17-dinitrocalix [4]arene (2). To a solution of diester 1 (3.93 g, $6.6 \mathrm{mmol})$ and acetic acid $(13.6 \mathrm{~mL}, 235 \mathrm{mmol})$ in $\mathrm{CH}_{2} \mathrm{Cl}_{2}(100$ $\mathrm{mL})$ was added $65 \% \mathrm{HNO}_{3}(23.3 \mathrm{~mL}, 335 \mathrm{mmol})$ at $0^{\circ} \mathrm{C}$. The reaction mixture was stirred at this temperature for $15 \mathrm{~min}$, whereupon water $(100 \mathrm{~mL})$ was added. The organic layer was separated, washed with water $(3 \times 100 \mathrm{~mL})$, and evaporated to give product 2 as a yellow solid after recrystallization from toluene: yield 51\%; mp 242-244 ${ }^{\circ} \mathrm{C}$ (toluene); ${ }^{1} \mathrm{H}$ NMR $\delta 8.97$ (s, $2 \mathrm{H}$ ), $8.02(\mathrm{~s}, 4 \mathrm{H}), 7.00$ (d, $4 \mathrm{H}, J=7.8 \mathrm{~Hz}), 6.87(\mathrm{t}, 2 \mathrm{H}, J=7.8$ $\mathrm{Hz}), 4.71(\mathrm{~s}, 4 \mathrm{H}), 4.50,3.53(\mathrm{~d}, 8 \mathrm{H}, J=13.4 \mathrm{~Hz}), 4.44(\mathrm{q}, 4 \mathrm{H}$ $J=7.0 \mathrm{~Hz}), 1.31(\mathrm{t}, 6 \mathrm{H}, J=7.0 \mathrm{~Hz}) ;{ }^{13} \mathrm{C} \mathrm{NMR} \delta 168.7,159.2$, $152.2,139.9,131.8(\mathrm{~s}), 129.9,129.0$ (d), $128.2(\mathrm{~s}), 125.3(\mathrm{~d}), 72.4$, 61.8, 31.3(t), 14.2 (q); MS-FAB m/z 687.6 [(M + H $)^{+}$, calcd 687.7]. Anal. Calcd for $\mathrm{C}_{36} \mathrm{H}_{34} \mathrm{~N}_{2} \mathrm{O}_{12}$ : $\mathrm{C}, 62.97 ; \mathrm{H}, 4.99 ; \mathrm{N}, 4.08$. Found: C, $62.80 ; \mathrm{H}, 5.24 ; \mathrm{N}, 3.84$.

25,26,27,28-Tetrakis[(ethoxycarbonyl)methoxy]-5,17-dinitrocalix[4]arene (3). A mixture of calix[4]arene 2 (3.5 g, 5.1 $\mathrm{mmol}), \mathrm{Na}_{2} \mathrm{CO}_{3}(5.6 \mathrm{~g}, 53 \mathrm{mmol})$, and bromoethyl acetate $(5.7$ $\mathrm{mL}, 51 \mathrm{mmol})$ in acetonitrile $(150 \mathrm{~mL})$ was refluxed for $48 \mathrm{~h}$. After filtration the solvent was removed, and the residue was dissolved in $\mathrm{CH}_{2} \mathrm{Cl}_{2}(100 \mathrm{~mL})$ and vigorously stirred with water for $15 \mathrm{~h}$ in order to remove sodium salts. After evaporation of $\mathrm{CH}_{2} \mathrm{Cl}_{2}$ the crude product was recrystallized from MeOH to give pure 3 as a colorless solid: yield $70 \% ; \mathrm{mp} 180^{\circ} \mathrm{C}(\mathrm{EtOH}) ;{ }^{1} \mathrm{H}$ NMR $\delta 7.57(\mathrm{~s}, 4 \mathrm{H}), 6.7-6.5(\mathrm{~m}, 6 \mathrm{H}), 4.93,3.35(\mathrm{~d}, 8 \mathrm{H}, J=13.9$ $\mathrm{Hz}), 4.86,4.63(\mathrm{~s}, 8 \mathrm{H}), 4.20(\mathrm{q}, 8 \mathrm{H}, J=7.0 \mathrm{~Hz}), 1.29(\mathrm{t}, 12 \mathrm{H}$, $J=7.0 \mathrm{~Hz}) ;{ }^{13} \mathrm{C}$ NMR $\delta 169.7,169.4,161.3,161.3,155.5,143.0$ (s), 136.3, 133.2(d), 129.2(s), 123.9(d), 71.5, 71.3,70.0,60.8, 31.4 (t), 14.2, 14.1 (q); MS-FAB $m / z$ 859.3 [(M $+\mathrm{H})^{+}$, calcd 859.3]. Anal. Calcd for $\mathrm{C}_{44} \mathrm{H}_{46} \mathrm{~N}_{2} \mathrm{O}_{16} \mathrm{CH}_{3} \mathrm{OH}: \mathrm{C}, 60.67 ; \mathrm{H}, 5.66 ; \mathrm{N}, 3.14$. Found: C, $60.80 ; \mathrm{H}, 5.34 ; \mathrm{N}, 3.14$

5,17-Diamino-25,26,27,28-tetrakis[(ethoxycarbonyl)methoxy]calix [4]arene (4b). A solution of 1,3-dinitro tetraester 3 $(3.4 \mathrm{~g}, 4 \mathrm{mmol})$ and $\mathrm{SnCl}_{2} \cdot 2 \mathrm{H}_{2} \mathrm{O}(8.9 \mathrm{~g}, 40 \mathrm{mmol}$ ) in ethanol (100 $\mathrm{mL}$ ) was refluxed for $6 \mathrm{~h}$. After the reaction mixture was poured onto ice it was adjusted to $\mathrm{pH} 8$. After extraction with $\mathrm{CH}_{2} \mathrm{Cl}_{2}$ $(2 \times 100 \mathrm{~mL})$, the organic layer was stirred with water for $5 \mathrm{~h}$. Evaporation of the solvent gave $\mathbf{4 b}$ as an orange oil: yield $55 \%$; ${ }^{1} \mathrm{H}$ NMR $\delta 6.70-6.50(\mathrm{~m}, 6 \mathrm{H}), 5.99(\mathrm{~s}, 4 \mathrm{H}), 5.83,3.12(\mathrm{~d}, 8 \mathrm{H}$, $J=13.9 \mathrm{~Hz}), 4.72,4.63(\mathrm{~s}, 8 \mathrm{H}), 4.20(\mathrm{q}, 8 \mathrm{H}, J=7.0 \mathrm{~Hz}), 3.21$ (br s, $4 \mathrm{H}), 1.30(\mathrm{t}, 12 \mathrm{H}, J=7.0 \mathrm{~Hz}) ;{ }^{13} \mathrm{C}$ NMR $\delta 170.4,170.3$, 156.0, 149.2, 141.3, 135.2, 134.7 (s), 128.5, 122.7, 115.8 (d), 71.5, $71.2,60.5,60.4,31.5(\mathrm{t}), 14.2(\mathrm{q}) ; \mathrm{MS}-\mathrm{FAB} m / z 799.3\left[(\mathrm{M}+\mathrm{H})^{+}\right.$ calcd 799.3]. Anal. Calcd for $\mathrm{C}_{44} \mathrm{H}_{60} \mathrm{~N}_{2} \mathrm{O}_{12} \cdot 0.25 \mathrm{CH}_{2} \mathrm{Cl}_{2}$ : $\mathrm{C}, 64.79$; $\mathrm{H}, 6.16 ; \mathrm{N}, 3.41$. Found: $\mathrm{C}, 64.42 ; \mathrm{H}, 5.93 ; \mathrm{N}, 3.34$.

General Procedure for the Preparation of 5a,b. Chloroacetyl chloride ( $1.6 \mathrm{~mL}, 20 \mathrm{mmol}$ ) was added dropwise to a solution of 1,3-diamino compound $4 \mathrm{a}, \mathrm{b}(10 \mathrm{mmol})$ and $\mathrm{Et}_{3} \mathrm{~N}(2.8$ $\mathrm{mL}, 20 \mathrm{mmol})$ in $\mathrm{CH}_{2} \mathrm{Cl}_{2}(75 \mathrm{~mL})$ at $\mathrm{rt}$. After the reaction mixture was stirred for $1 \mathrm{~h}$, the organic layer was washed with $0.5 \mathrm{~N} \mathrm{HCl}$ $(2 \times 50 \mathrm{~mL})$ and water $(2 \times 50 \mathrm{~mL})$ and evaporated. Column chromatography [neutral $\mathrm{Al}_{2} \mathrm{O}_{3}$ (activity I), ethyl acetate] gave pure $\mathbf{5 a}, \mathbf{b}$.

5,17-Bis(2-chloroacetamido)-25,26,27,28-tetrapropoxycalix[4]arene (5a): yield $69 \%$; mp $155-157^{\circ} \mathrm{C}$ (ethyl acetate petroleum ether); ${ }^{1} \mathrm{H}$ NMR $\delta 7.94(\mathrm{br} \mathrm{s}, 2 \mathrm{H}), 6.69(\mathrm{~s}, 4 \mathrm{H}), 6.6-6.4$ $(\mathrm{m}, 6 \mathrm{H}), 4.43,3.12(\mathrm{~d}, 8 \mathrm{H}, J=13.9 \mathrm{~Hz}), 4.10(\mathrm{~s}, 4 \mathrm{H}), 3.9-3.7$ $(\mathrm{m}, 8 \mathrm{H}), 2.0-1.6(\mathrm{~m}, 8 \mathrm{H}), 1.05(\mathrm{t}, 12 \mathrm{H}, J=7.2 \mathrm{~Hz}) ;{ }^{13} \mathrm{C}$ NMR $\delta 170.0,153.6,137.7,134.2,130.8,129.2$ (s), 128.7, 122.2, 121.3

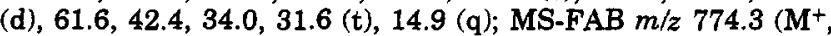

calcd 774.3). Anal. Calcd for $\mathrm{C}_{44} \mathrm{H}_{62} \mathrm{Cl}_{2} \mathrm{~N}_{2} \mathrm{O}_{6}: \mathrm{C}, 68.12 ; \mathrm{H}, 6.76$; $\mathrm{N}, 3.61$. Found: C, $68.00 ; \mathrm{H}, 6.57 ; \mathrm{N}, 3.59$.

5,17-Bis(2-chloroacetamido)-25,26,27,28-tetrakis[(ethoxycarbonyl)methoxy]calix[4]arene (5b): yield $64 \%$; mp $190-192{ }^{\circ} \mathrm{C}$ (ethyl acetate/petroleum ether); ${ }^{1} \mathrm{H}$ NMR $\delta 8.10$ (br $\mathrm{s}, 2 \mathrm{H}), 6.85(\mathrm{~s}, 4 \mathrm{H}), 6.7-6.6(\mathrm{~m}, 6 \mathrm{H}), 4.89,3.23(\mathrm{~d}, 8 \mathrm{H}, J=13.5$ $\mathrm{Hz}), 4.71,4.69(\mathrm{~s}, 8 \mathrm{H}), 4.21(\mathrm{q}, 8 \mathrm{H}, J=7.0 \mathrm{~Hz}), 4.09(\mathrm{~s}, 4 \mathrm{H})$, $1.25(\mathrm{t}, 12 \mathrm{H}, J=7.0 \mathrm{~Hz}) ;{ }^{13} \mathrm{C}$ NMR $\delta 170.0,163.7,155.6,153.5$, $135.5,134.0,131.2,129.2$ (s), 128.8, 123.2, 121.3 (d), 71.4, 71.3, 60.6, 42.4, 31.5 (t), 14.2 (q); MS-FAB m/z 950.3 (M+ , calcd 950.3). Anal. Calcd for $\mathrm{C}_{48} \mathrm{H}_{52} \mathrm{Cl}_{2} \mathrm{~N}_{2} \mathrm{O}_{14}: \mathrm{C}, 60.61 ; \mathrm{H}, 5.47 ; \mathrm{N}, 2.94$. Found: C, $60.65 ; \mathrm{H}, 5.65 ; \mathrm{N}, 2.67$.

General Procedure for the Preparation of 6a,b. A mixture of $5 \mathbf{a}, \mathbf{b}(1 \mathrm{mmol}), 2$-(2-allyloxy)-3-hydroxybenzaldehyde ${ }^{12}(0.36$ $\mathrm{g}, 2 \mathrm{mmol}), \mathrm{K}_{2} \mathrm{CO}_{3}(0.28 \mathrm{~g}, 2 \mathrm{mmol})$, and potassium iodide $(0.17$ $\mathrm{g}, 1 \mathrm{mmol})$ in acetonitrile $(150 \mathrm{~mL})$ was refluxed for $12 \mathrm{~h}$. After filtration the solvent was removed and the crude product was purified by column chromatography [neutral $\mathrm{Al}_{2} \mathrm{O}_{3}$ (activity I), ethyl acetate].

5,17-Bis[[3-formyl-2-(2-propenyloxy)phenoxy]acetamido]25,26,27,28-tetrapropoxycalix[4] arene (6a): yield $59 \%$; $\mathrm{mp}$ 100-101 ${ }^{\circ} \mathrm{C}\left(\mathrm{CH}_{2} \mathrm{Cl}_{2}\right.$ petroleum ether); ${ }^{1} \mathrm{H}$ NMR $\delta 10.34(\mathrm{~s}, 2 \mathrm{H})$ $8.14(\mathrm{br} \mathrm{s}, 2 \mathrm{H}), 7.39$ (d, $2 \mathrm{H}, J=7.5 \mathrm{~Hz}), 7.2-7.0(\mathrm{~m}, 4 \mathrm{H}), 6.94$ $(\mathrm{s}, 4 \mathrm{H}), 6.6-6.4(\mathrm{~m}, 6 \mathrm{H}), 6.1-6.0(\mathrm{~m}, 2 \mathrm{H}), 5.5-5.0(\mathrm{~m}, 4 \mathrm{H})$ $4.6-4.5(\mathrm{~m}, 4 \mathrm{H}), 4.53(\mathrm{~s}, 4 \mathrm{H}), 4.50,3.16(\mathrm{~d}, 8 \mathrm{H}, J=13.7 \mathrm{~Hz})$ 3.85 ( $, 8 \mathrm{H}, J=7.0 \mathrm{~Hz}$ ), 2.0-1.8 (m, $8 \mathrm{H}), 1.00,0.95(\mathrm{t}, 12 \mathrm{H}$ $J=7.0 \mathrm{~Hz}) ;{ }^{13} \mathrm{C}$ NMR $\delta 189.3$ (d), 164.9, 156.3, 154.1, 151.1, $150.5,135.8,134.6$ (s), 132.7 (d), 130.6, 130.3 (s), 128.2, 125.0 $122.3,121.5,120.9,120.1$ (d), 119.0, 76.8, 76.1, 68.8, 31.1, 23.2, $23.1(\mathrm{t}), 10.3,10.2(\mathrm{q})$; MS-FAB $\mathrm{m} / z$ 1059.2 ( $\mathrm{M}^{+}$, calcd 1059.3) Anal. Calcd for $\mathrm{C}_{64} \mathrm{H}_{70} \mathrm{~N}_{2} \mathrm{O}_{12}: \mathrm{C}, 72.57 ; \mathrm{H}, 6.66 ; \mathrm{N}, 2.64$. Found: C, 72.36; H, 6.55; N, 2.56.

25,26,27,28-Tetrakis[(ethoxycarbonyl)methoxy]-5,17-bis[3-formyl-2-[(2-propenyloxy)phenoxy]acetamido]calix [4] arene (6b): yield $64 \% ; \mathrm{mp} 69-71{ }^{\circ} \mathrm{C}\left(\mathrm{CH}_{2} \mathrm{Cl}_{2}\right.$ /petroleum ether) ${ }^{1} \mathrm{H}$ NMR $\delta 10.33(\mathrm{~s}, 2 \mathrm{H}), 8.29(\mathrm{br} \mathrm{s}, 2 \mathrm{H}), 7.43(\mathrm{~d}, 2 \mathrm{H}, J=7.5$ $\mathrm{Hz}), 7.2-7.0(\mathrm{~m}, 4 \mathrm{H}), 6.99(\mathrm{~s}, 4 \mathrm{H}), 6.7-6.5(\mathrm{~m}, 6 \mathrm{H}), 6.1-6.0(\mathrm{~m}$ $2 \mathrm{H}), 5.5-5.1(\mathrm{~m}, 4 \mathrm{H}), 4.91,3.25(\mathrm{~d}, 8 \mathrm{H}, J=13.4 \mathrm{~Hz}), 4.76,4.74$ $(\mathrm{s}, 8 \mathrm{H}), 4.65-4.60(\mathrm{~m}, 4 \mathrm{H}), 4.54(\mathrm{~s}, 4 \mathrm{H}), 4.22(\mathrm{q}, 8 \mathrm{H}, J=7.0$ $\mathrm{Hz}), 1.29\left(\mathrm{t}, 12 \mathrm{H}, J=7.0 \mathrm{~Hz}\right.$ ); ${ }^{13} \mathrm{C}$ NMR $\delta 189.3$ (d), $170.1,170.0$, $165.0,155.6,153.3,151.1,150.8,135.5,134.0$ (s), 132.7 (d), 131.2, 130.6 (s), 128.6, 125.1, 123.2, 121.6, 121.2, 120.2 (d), 118.9, 76.2, $71.4,68.8,60.6,31.6(\mathrm{t}), 14.2(\mathrm{q})$; MS-FAB $m / z 1235.4\left(\mathrm{M}^{+}\right.$, calcd 1235.3). Anal. Calcd for $\mathrm{C}_{68} \mathrm{H}_{70} \mathrm{~N}_{2} \mathrm{O}_{20}: \mathrm{C}, 66.12 ; \mathrm{H}, 5.71 ; \mathrm{N}, 2.27$. Found: C, 65.97; H, 5.82; N, 2.34 .

General Procedure for the Deallylation ${ }^{13}$ of Aldehydes 6a,b. Formation of Aldehydes 7a,b. A mixture of $6 \mathbf{a}, \mathbf{b}$ ( 3 $\mathrm{mmol}$ ), $\mathrm{Pd}(\mathrm{OAc})_{2}(20 \mathrm{mg}, 0.1 \mathrm{mmol}), \mathrm{PPh}_{3}(125 \mathrm{mg}, 0.5 \mathrm{mmol}$ ) $\mathrm{Et}_{3} \mathrm{~N}(3.7 \mathrm{~g}, 37 \mathrm{mmol})$, and $\mathrm{HCOOH}(1.65 \mathrm{~g}, 37 \mathrm{mmol})$ in $80 \%$ aqueous EtOH $(60 \mathrm{~mL})$ was refluxed for $1 \mathrm{~h}$. The solvent was evaporated, and the total water volume was adjusted at $100 \mathrm{~mL}$ The product was extracted with $\mathrm{CH}_{2} \mathrm{Cl}_{2}(3 \times 100 \mathrm{~mL})$ and washed with water $(2 \times 100 \mathrm{~mL})$. The solvent was removed to give $\mathbf{7 a , b}$ as yellow oils which were used without purification due to slow decomposition.

5,17-Bis[(3-formyl-2-hydroxyphenoxy)acetamido]-25,26,27,28-tetrapropoxycalix [4] arene (7a): yield 79\%; ${ }^{1} \mathrm{H}$ NMR $\delta$ $9.91(\mathrm{~s}, 2 \mathrm{H}), 8.90(\mathrm{br} \mathrm{s}, 2 \mathrm{H}), 7.1-6.5(\mathrm{~m}, 16 \mathrm{H}), 4.41(\mathrm{~s}, 4 \mathrm{H}), 4.37$, $3.17(\mathrm{~d}, 8 \mathrm{H}, J=13.4 \mathrm{~Hz}), 3.8-3.6(\mathrm{~m}, 8 \mathrm{H}), 2.1-1.9(\mathrm{~m}, 8 \mathrm{H})$, $1.0-0.9(\mathrm{~m}, 12 \mathrm{H}) ;$ MS-FAB $\mathrm{m} / \mathrm{z} 978.3\left[(\mathrm{M}+\mathrm{H})^{+}\right.$, calcd for $\left.\mathrm{C}_{58} \mathrm{H}_{60} \mathrm{~N}_{2} \mathrm{O}_{12} 978.1\right]$.

25,26,27,28-Tetrakis[(ethoxycarbonyl)methoxy]-5,17-bis[(3-formyl-2-hydroxyphenoxy)acetamido]calix [4]arene (7b): yield 84\%; ${ }^{1} \mathrm{H}$ NMR $\delta 9.93(\mathrm{~s}, 2 \mathrm{H}), 8.62(\mathrm{br} \mathrm{s}, 2 \mathrm{H}), 7.27$ $(\mathrm{d}, 2 \mathrm{H}, J=7.5 \mathrm{~Hz}), 7.20,7.17$ (d, $4 \mathrm{H}, J=7.5 \mathrm{~Hz}), 6.9-6.8(\mathrm{~m}$, $6 \mathrm{H}), 6.53(\mathrm{~s}, 4 \mathrm{H}), 4.96,3.30(\mathrm{~d}, 8 \mathrm{H}, J=13.4 \mathrm{~Hz}), 4.81,4.74,4.42$ $(\mathrm{s}, 12 \mathrm{H}), 4.25-4.20(\mathrm{~m}, 8 \mathrm{H}), 1.34(\mathrm{t}, 12 \mathrm{H}, J=7.0 \mathrm{~Hz})$; MS-FAB $m / z 1154.4\left[(\mathrm{M}-\mathrm{H})^{-}\right.$, calcd for $\left.\mathrm{C}_{62} \mathrm{H}_{62} \mathrm{~N}_{2} \mathrm{O}_{20} 1154.1\right]$.

General Procedure for the Synthesis of $\mathrm{UO}_{2}-$ Salenes $8 \mathbf{a}, \mathbf{b}$. Solutions of bisaldehydes $7 \mathbf{a}, \mathbf{b}(1.3 \mathrm{mmol})$ and cis-1,2cyclohexanediamine $(0.14 \mathrm{~g}, 1.3 \mathrm{mmol})$ in $\mathrm{EtOH}(50 \mathrm{~mL})$ were added separately to a refluxing solution of $\mathrm{UO}_{2}(\mathrm{OAc})_{2} \cdot 2 \mathrm{H}_{2} \mathrm{O}(0.56$ $\mathrm{g}, 1.3 \mathrm{mmol}$ ) in $\mathrm{EtOH}(500 \mathrm{~mL}$ ) for $2 \mathrm{~h}$, whereupon refluxing was continued for $1 \mathrm{~h}$. After the solution was cooled, the solvent was evaporated. The residue was dissolved in $\mathrm{CH}_{2} \mathrm{Cl}_{2}(150 \mathrm{~mL})$ and stirred with water for $15 \mathrm{~h}$. After evaporation of $\mathrm{CH}_{2} \mathrm{Cl}_{2}$ the crude mixture was purified by column chromatography $\left(\mathrm{SiO}_{2}\right.$, $\mathrm{CH}_{2} \mathrm{Cl}_{2}$ /ethyl acetate, $5: 1$ ) to give $\mathbf{8 a}, \mathbf{b}$ as orange solids. 
5,17-[[[2,2'-[1,2-Cyclohexanediylbis[nitrilomethyl(2-hydroxy-3,1-phenylene)oxy]]bis(acetamido)](2-)]dioxouranium]-25,26,27,28-tetrapropoxycalix [4] arene (8a): yield 9\%; mp 283-285 ${ }^{\circ} \mathrm{C}$ (acetonitrile); ${ }^{1} \mathrm{H}$ NMR (DMSO- $\left.d_{6} / \mathrm{CDCl}_{3}, 8: 1\right) \delta$ $9.45(\mathrm{br} \mathrm{s}, 2 \mathrm{H}), 9.34(\mathrm{~s}, 2 \mathrm{H}), 7.31,7.26(\mathrm{~d}, 4 \mathrm{H}, J=8.0 \mathrm{~Hz})$, $7.1-6.8(\mathrm{~m}, 10 \mathrm{H}), 6.45(\mathrm{t}, 2 \mathrm{H}, J=8.0 \mathrm{~Hz}), 4.81(\mathrm{q}, 4 \mathrm{H}, J=7.0$ $\mathrm{Hz}), 4.55-4.50(\mathrm{~m}, 2 \mathrm{H}), 4.30,3.12(\mathrm{~d}, J=13.5 \mathrm{~Hz}), 4.00,3.61$ $(\mathrm{t}, 8 \mathrm{H}, J=7.0 \mathrm{~Hz}), 2.3-2.0(\mathrm{~m}, 8 \mathrm{H}), 1.00,0.97(\mathrm{t}, 12 \mathrm{H}, J=7.0$ $\mathrm{Hz}$ ); MS-FAB m/z 1325.7 ( $\mathrm{M}^{+}$, calcd 1325.3). Anal. Calcd for $\mathrm{C}_{64} \mathrm{H}_{70} \mathrm{~N}_{4} \mathrm{O}_{12} \mathrm{U} \cdot \mathrm{CH}_{3} \mathrm{CN}$ : C, 58.02; $\mathrm{H}, 5.39 ; \mathrm{N}$, 5.13. Found: $\mathrm{C}$, $58.32 ; \mathrm{H}, 5.56 ; \mathrm{N}, 5.15$.

5,17-[[[2,2'-[1,2-Cycloheranediylbis[nitrilomethyl(2-hy droxy-3,1-phenylene)oxy]]bis(acetamido)](2-)]dioxouranium]-25,26,27,28-tetrakis [(ethoxycarbonyl)methoxy]calix[4]arene (8b): yield $15 \%$; mp $235-238^{\circ} \mathrm{C}$ (EtOH); ${ }^{1 \mathrm{H}} \mathrm{NMR}$ $\left(\mathrm{DMSO}-d_{6}\right) \delta 9.70$ (br 8, $2 \mathrm{H}$ ), $9.48(\mathrm{~s}, 2 \mathrm{H}), 7.43,7.36(\mathrm{~d}, 4 \mathrm{H}, J$
$=8.0 \mathrm{~Hz}), 7.2-6.9(\mathrm{~m}, 10 \mathrm{H}), 6.65(\mathrm{t}, 2 \mathrm{H}, J=8.0 \mathrm{~Hz}), 5.24(\mathrm{~s}$, $4 \mathrm{H}), 4.80,3.19(\mathrm{~d}, 8 \mathrm{H}, J=13.6 \mathrm{~Hz}), 4.69,4.46(\mathrm{~s}, 8 \mathrm{H}), 4.7-4.6$ (m, $2 \mathrm{H}), 4.25,4.10(\mathrm{q}, 8 \mathrm{H}, J=7.0 \mathrm{~Hz}), 2.4-2.3(\mathrm{~m}, 2 \mathrm{H}), 1.9-1.6$ $(\mathrm{m}, 6 \mathrm{H}), 1.30,1.21(\mathrm{t}, 12 \mathrm{H}, J=7.0 \mathrm{~Hz}) ; \mathrm{MS}-\mathrm{FAB} \mathrm{m} / \mathrm{z} 1501.1\left(\mathrm{M}^{+}\right.$, calcd 1501.3). Anal. Calcd for $\mathrm{C}_{68} \mathrm{H}_{70} \mathrm{~N}_{4} \mathrm{O}_{20} \mathrm{U}: \mathrm{C}, 54.40 ; \mathrm{H}, 4.70$; $\mathrm{N}, 3.73$. Found: C, 54.39; H, 4.86; N, 3.55.

Determination of Association Constants. The measurements were performed by ${ }^{1} \mathrm{H}$ NMR titration experiments in DMSO- $d_{6}$ at $298 \mathrm{~K}$ using a constant host concentration of $4 \mathrm{mM}$ and a varying guest concentration of $0.3-30 \mathrm{mM}$. As a probe the chemical shift of the C(O)NH signal was used. The $K_{\text {ans }}$ values were calculated by nonlinear regression as described in ref 19 . The estimated error is $<5 \%$.

(19) de Boer, J. A. A.; Reinhoudt, D. N.; Harkema, S.; van Hummel, G. J.; de Jong, F. J. Am. Chem. Soc. 1982, 104, 4073. 